Reporte de casos 


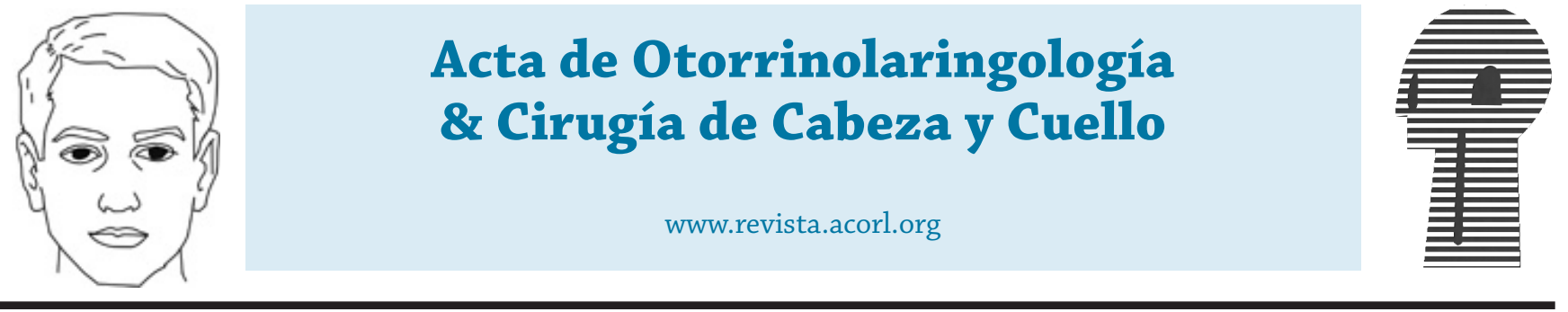

\title{
Sialolitiasis submandibular gigante, extracción quirúrgica y reparación ductal: reporte de caso.
}

\section{Giant submandibular sialolithiasis, surgical removal and ductal repair: case report.}

\author{
Christian Kammerer*, Diego Escobar**, Jorge Holguín*** \\ * Otorrinolaringólogo, Universidad del Valle - Hospital Universitario del Valle. Cali, Colombia. \\ ** Docente de otorrinolaringología, Universidad del Valle - Hospital Universitario del Valle. Cali, Colombia. \\ *** Docente de otorrinolaringología, Universidad del Valle - Hospital Universitario del Valle. Cali, Colombia.
}

Forma de citar: Kammerer C, Escobar D, Holguín J. Sialolitiasis submandibular gigante, extracción quirúrgica y reparación ductal: reporte de caso. Acta otorrinolaringol. cir. cabeza cuello. 2020;48(4): 311-314 Doi: 10.37076/acorl.v48i4.352

\section{INFORMACIÓN DEL ARTÍCULO}

Historia del artículo:

Recibido: 17 de Septiembre 2018

Evaluado: 9 de Noviembre de 2020

Aceptado: 27 de Noviembre de 2020

Palabras clave (DeCS):

Cálculos del conducto salival;

glándula submandibular,

\section{RESUMEN}

Introducción: la patología litiásica de las glándulas salivales forma parte de un grupo de afecciones que perjudica el sistema de drenaje normal de la saliva hacia la cavidad oral, ocasionando lesiones múltiples sobre la glándula afectada. Se realizó el reporte de un caso clínico con esta patología que, por el tamaño del lito, se considera extremadamente rara en nuestro medio. Método: se realizó el reporte de caso y revisión de la literatura (estudios radiológicos, historia clínica y hallazgos clínicos); además, el paciente firmó el consentimiento para esta publicación. Resultados: un paciente masculino de 38 años remitido de consulta externa con un cuadro clínico de 1 semana de dolor, edema y tumefacción en la región submandibular izquierda asociado con una secreción sialopurulenta por la cavidad oral. En la radiografía ( $\mathrm{Rx})$ oclusal estricta y en la tomografía axial computarizada (TAC) de cuello contrastado se evidenció una lesión litiásica de más o menos 4,5 x 1,8 cm de diámetro, de característica radiolúcida, a nivel del conducto de wharton. Se dio un manejo quirúrgico por otorrinolaringología maxilofacial, consistente en sialolitotomía y antibioticote- 
Key words (MeSH):

Salivary duct stones; submandibular gland, rapia por 10 días, con las cuales se obtuvieron buenos resultados. Conclusiones: la litiasis gigante a nivel del sistema salival (glándula, conductos) es una patología poco común en nuestro medio y está involucrada en el desarrollo de múltiples patología de tipo inflamatorio recurrente de la glándula involucrada, lo cual afecta la calidad de vida de los pacientes. Por esta razón, el diagnóstico y manejo debe ser oportuno para evitar complicaciones, como una infección profunda del cuello; en nuestro caso, el manejo fue oportuno con una extracción quirúrgica por la vía oral del cálculo y la reparación del conducto de wharton del lado izquierdo.

\section{ABSTRACT}

Introduction: the lithiasic pathology of the salivary glands is part of a group of conditions that harms the normal drainage system of the saliva towards the oral cavity, causing multiple lesions on the affected gland, a report of a clinical case with this pathology was made. Due to the size of the stone it is considered extremely rare in our environment. Method: case report and review of the literature (radiological studies, clinical history, clinical findings) patient consent was signed for this publication. Results: a 38-year-old male patient referred from the outpatient clinic with a 1-week clinical picture of pain, swelling and edema in the left submandibular region associated with sialopurulent secretion from the oral cavity in the strict occlusal $\mathrm{Rx}$ and a contrast-enhanced neck CT revealed a lithiasic lesion with more or less $4.5 \mathrm{x}$ $1.8 \mathrm{~cm}$ in diameter of radiolucent features at the level of the wharton duct, surgical management was given by maxillofacial ENT, consisting of sialolitotomy and antibiotic therapy for 10 days with good results. Conclusions: the giant lithiasis at the level of the salivary system (gland, ducts) is a rare pathology in our environment, is involved in the development of multiple pathologies of recurrent inflammatory type of the affected gland, affecting the quality of life of patients, so the diagnosis and management should be timely and avoid complications, such as deep neck infection; in our case, the management was timely with oral surgical removal of the calculus and repair of the wharton duct on the left side.

\section{Introducción}

La sialolitiasis se define como una patología caracterizada por la presencia de un lito/piedra que causa una obstrucción en el drenaje de una glándula salival y/o de su conducto excretor. Teóricamente, los litos en las glándulas y los conductos salivales se originan por el acumulo de mineral de diferentes composiciones que rodea a un cuerpo extraño, detritus celulares, microorganismos, etc. Esta patología puede estar asociada con procesos infecciosos e inflamatorios que originan signos y síntomas verdaderamente incapacitantes.

\section{Caso clínico}

Presentamos el caso clínico de un paciente masculino de 38 años remitido de consulta externa con un cuadro clínico de una semana de evolución dado por edema, dolor y tumefacción a nivel submandibular izquierdo, asociado con una secreción sialopurulenta por la cavidad oral, lo que dificultaba su alimentación debido a una alteración en la masticación. Como antecedentes tenemos litiasis renal, negó traumas previos y/o procesos infecciosos antiguos a nivel de la cavidad oral.

En el examen físico se observó un edema del piso de la boca de predominio izquierdo y se palpó una masa de consis- tencia pétrea de más o menos 4 x 1,5 cm de diámetro, la cual obstruía completamente el conducto de wharton y desplazaba la glándula lateralmente. $\mathrm{Al}$ ejercer digitopresión, hubo una expulsión de material sialopurulento; no hay adenopatías palpables en el cuello.

El paciente ingresó a una institución de tercer nivel, donde se le realizó una radiografía oclusal estricta y una tomografía de cuello contrastado. En estas se observó una lesión litiásica de más o menos 4,5 x 1,8 cm de diámetro, de características radiolúcidas, que obstruía completamente el conducto de wharton del lado izquierdo, así como un aumento de volumen de la glándula submandibular sin puntos de necrosis, abscesos, ni crecimiento tumoral (Figuras 1-2-3).

Se inició el tratamiento antibiótico con ampicilina-sulbactam, un analgésico y medios locales. Finalmente, se llevó paciente a una extraccion quirúrgica del calculo, a nivel submandibular izquierdo y reparación del conducto, conservando la papila (Figuras 4-5).

El paciente toleró el procedimiento sin complicaciones. Se le realizó manejo ambulatorio con ampicilina-sulbactam oral por 10 días, se sugirió hidratación abundante y se le indicaron recomendaciones y signos de alarma por los cuales debían acudir al servicio de urgencias. Los controles posoperatorios respectivos fueron satisfactorios y no hubo ninguna sintomatología asociada. El nivel de paratohormona 


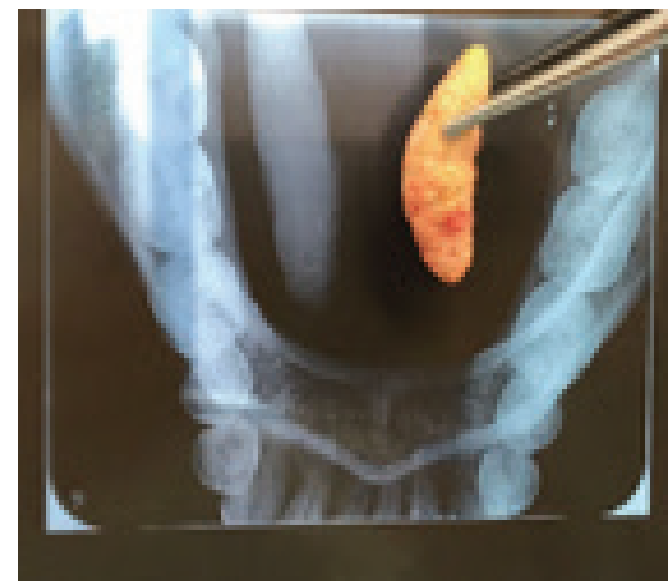

Figuras 1. Tomografía de cuello contrastado, comparando lesión lítica macroscópica

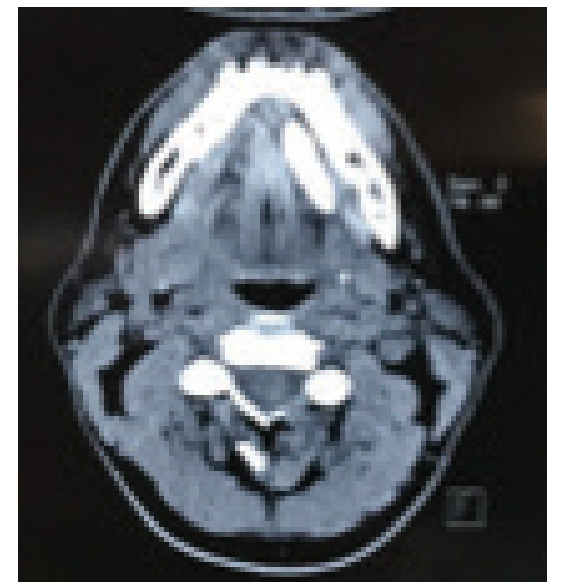

Figura 2. Radiografía oclusal estricta

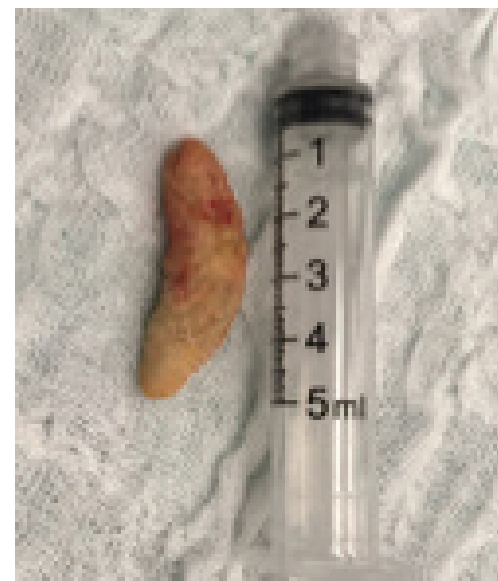

Figura 3. Lesión litiasica de $4,5 \times 1,8 \mathrm{~cm}$ aproximadamente

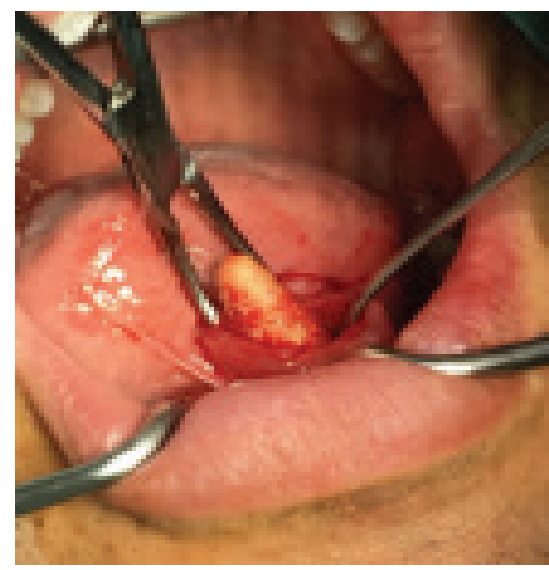

Figura 4. extracción quirúrgica de la lesión litiasica

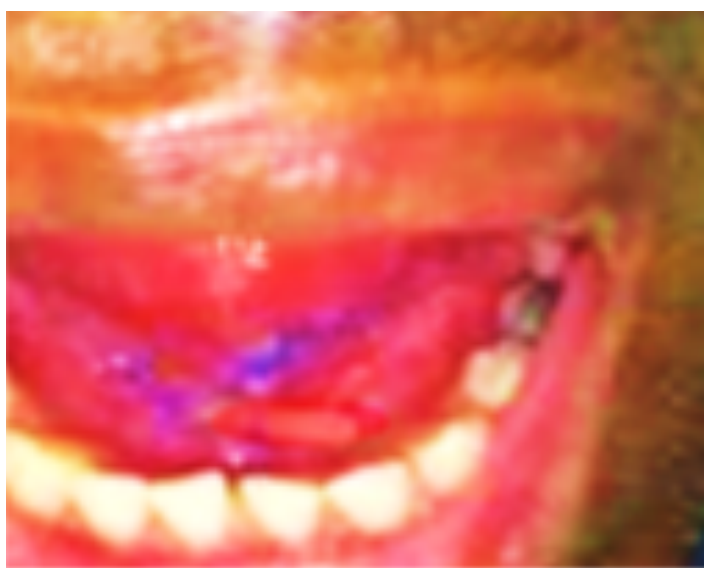

Figura 5. reparación del conducto, conservando la papila de ingreso fue de $58,25 \mathrm{pg} / \mathrm{mL}$ y el análisis del cálculo por espectro infrarrojo reportó un fosfato cálcico apatítico.

\section{Discusión}

La sialolitiasis es una condición clínica que se caracteriza por la obstrucción parcial o completa de tipo mecánico de una glándula salival o de su conducto excretor causada por la presencia de cálculos o sialolitos, lo que puede ocasionar una alteración en la fisiología de la glándula, sobreinfectarse y cronificarse, y ser altamente incapacitante para el paciente. Los sialolitos son formaciones calcáreas que pueden ser únicas o múltiples, con una variedad en su forma y composición (calcio, fósforo, magnesio, amonio); pueden ser ovalados o redondos y, por lo general, toman la forma del conducto. La etiología de este tipo de afección no se conoce con exactitud, por lo que existen varias hipótesis al respecto; todas concuerdan en que estos depósitos calcáreos se forman por la mineralización y la compactación de cuerpos extraños, células epiteliales y algunos microorganismos (1). Se piensa que afecta a 60 millones de personas por año; la sialolitiasis de la glándula submandibular es la más común (80-90 \%), seguida de la sialolitiasis de la glándula parótida (5-15 \%). No es común encontrar sialolitos múltiples en la misma glándula (2-4).

Inicialmente, se cree que se deposita contenido orgánico, a lo mejor glucoproteínas, que posteriormente inicia su mineralización. Se sugiere que puede ser secundario a traumatismos, que posiblemente dificultan el flujo salival y provocan estancamiento; por otra parte, la saliva hipersaturada facilita la formación del cálculo. Los sialolitos pueden aparecer a cualquier edad, pero el pico de máxima frecuencia se sitúa entre la cuarta y la sexta década de la vida (1). La obstrucción incompleta por un sialolito se asocia con una infección secundaria de la glándula ocasionada por una obstrucción completa y una atrofia glandular (2).

El diagnóstico diferencial debe hacerse con sialoadenitis, hipertrofia maseterina, patologías relacionadas con la articulación temporomandibular, osteomielitis, mucocele, quistes de retención mucoso, abscesos sublinguales y otras alteraciones del piso de la boca (1).

Las ayudas más comunes implementadas para el diagnóstico de la sialolitiasis son la sialografía convencional, la ecografía, la resonancia magnética nuclear, la tomografía en- 
doscopia y las radiografías oclusales, el $40 \%$ de los cálculos parotídeos y el $20 \%$ de los submandibulares no son radiopacos y necesitan sialografía para su diagnóstico. El manejo de estas alteraciones incluye procedimientos no quirúrgicos y quirúrgicos que implican, en ocasiones, la extracción de la glándula. Las complicaciones no infecciosas que se pueden presentar son un hematoma en el piso de la boca y la región submandibular, y una lesión de la rama marginal mandibular del nervio facial y nervio lingual; en la mayoría de los casos, estas lesiones se resuelven espontáneamente (3-6).

El tratamiento de la sialolitiasis se basa en conocer la ubicación y el tamaño del sialolito. Los pacientes se benefician del manejo conservador si el cálculo es pequeño. El paciente debe estar bien hidratado, aplicar calor húmedo y un masaje de la glándula, mientras que los sialogogos se usan para aumentar la producción de saliva para expulsar el calculo; La sialolitiasis con sobreinfección debe tratarse con antibióticos. La mayoría de los cálculos responden al tratamiento combinado de un antibiótico y la resección quirúrgica, cuando sea necesario $(2,5,7$, ). La sialoadenoscopia no requiere ser invasiva, ya que se realiza para los sialolitos grandes y la obliteración ductal, los calculos pequeños ubicados en el conducto se pueden eliminar mediante masaje e hidratación en una sola pieza o en fragmentos que se expulsan de forma intermitente. (9)

Cabe destacar que la utilización del láser de $\mathrm{CO} 2$, debido a sus ventajas de sangrado mínimo, menos cicatrices, visión clara y complicaciones posoperatorias mínimas, está ganando popularidad en el tratamiento de la sialolitiasis $(2,8)$.

\section{Conclusiones}

La sialolitiasis es una condición relativamente frecuente que puede ocasionar verdaderas complicaciones y comprometer la calidad de vida del paciente, principalmente en los episodios de sobreinfección. No es raro que estas complicaciones puedan causar infecciones profundas del cuello, potencialmente mortales, por lo que el diagnóstico y el tratamiento con antibióticos, analgésicos, sialogogos y resecciones qui- rúrgicas debe ser adecuado y oportuno. Han surgido varias modalidades avanzadas de diagnóstico y tratamiento en la sialolitiasis, pero las técnicas convencionales conservan su popularidad hasta la fecha; en nuestro caso, el paciente recibió manejo médico con antibióticos y manejo quirúrgico con la resección del lito, con una evolución favorable y una resolución completa de su patología (5).

\section{Conflicto de interés}

No presenta ningún conflicto de interés

\section{REFERENCIAS}

1. Rebolledo Cobos M, Carbonell Muñoz Z, Díaz Caballero A. Sialolitos en conductos y glándulas salivales: Revisión de literatura. Av Odontoestomatol. 2009;25(6):311-7.

2. Thopte S, Ul Nisa S, Jadhav A, Chaudhari R. Sialolithiasis of submandibular gland with acute suppurative sialadenitis: a case report. World Journal of Pharmacy and Pharmaceutical Sciences. 2016;5(4):X-X.

3. Khan M, Mehboob B, Ahmad T. A spectrum of the surgical management of sub-mandibular sialolithiasis — a study. Pakistan Oral \& Dental Journal. 2016;36(3):375-8.

4. Nezhad C, Mehravaran R, Sharafi M. Large Submandibular Sialoliths: A Report of Three Cases. Journal of Dentistry and Oral Care Medicine. 2016;2(1):1-6.

5. Tulasi Lakshmi D, Firoz Babu P, Negi LS, Nayyar AS, Recurrent Sialadenitis with Sialolithiasis of Submandibular Gland: A Case Report. J Dent App. 2016; 3(4): 358-360.

6. Shameeka thopte, sialolithiasis of submandibular gland with acute suppurative sialadenitis: a case report University Dental College and Hospital - Pune. 2016

7. Kaur H, Jain S, Kamboj R, Pandav G. Submandibular Salivary Sialolith: A Case Report with Review of Literature. OHDM. 2016;15(1):18-21.

8. Bakshi SS. A Hard Calculus: Submandibular Sialolithiasis. Am J Med. 2017;130(2):161-162.

9. a hard calculus: submandibular sialolithiasis: departament of ear, nose and head and neck india; satvinder sing bakshi -elsevier 2016 